\title{
Energy Reflection and Transmission at 2D Holographic Interfaces
}

\author{
Constantin Bachas $\odot,{ }^{1, *}$ Shira Chapman $\odot,{ }^{2, \dagger}$ Dongsheng Ge $\odot,{ }^{1,3}$ and Giuseppe Policastro $\oplus^{1, \S}$ \\ ${ }^{1}$ Laboratoire de Physique de l'École Normale Supérieure, CNRS, PSL Research University and Sorbonne Universités, \\ 24 rue Lhomond, 75005 Paris, France \\ ${ }^{2}$ Institute for Theoretical Physics, University of Amsterdam, \\ Science Park 904, Postbus 94485, 1090 GL Amsterdam, The Netherlands \\ ${ }^{3}$ Kavli Institute for Theoretical Physics, University of California, Santa Barbara, California 93106, USA
}

(Received 10 July 2020; revised 26 August 2020; accepted 28 October 2020; published 2 December 2020)

\begin{abstract}
Scattering from conformal interfaces in two dimensions is universal in that the flux of reflected and transmitted energy does not depend on the details of the initial state. In this Letter, we present the first gravitational calculation of energy reflection and transmission coefficients for interfaces with thin-brane holographic duals. Our result for the reflection coefficient depends monotonically on the tension of the dual string anchored at the interface and obeys the lower bound recently derived from the averaged-null-energy condition in conformal field theory. The boundary-conformal-field-theory limit is recovered for infinite ratio of the central charges.
\end{abstract}

DOI: 10.1103/PhysRevLett.125.231602

Introduction.-Conformal interfaces are ubiquitous both in condensed-matter systems and in studies of the holographic duality. Such interfaces describe the local, scaleinvariant gluing of two conformal field theories, $\mathrm{CFT}_{L}$ on the left and $\mathrm{CFT}_{R}$ on the right. Examples include junctions of quantum wires [1], line or surface defects in the critical $2 \mathrm{D}$ or 3D Ising models [2], or the gluing of superconformal gauge theories with different couplings and/or gauge groups. In bottom-up AdS/CFT, interfaces are often modeled by codimension-one branes anchored at the anti-de Sitter (AdS) boundary. Smooth (super)gravity solutions describing top-down embeddings in string theory are also known. Some early papers on the subject are Refs. [3-8].

Folding spacetime along an interface converts the latter to a conformal boundary of the product theory $\mathrm{CFT}_{L} \otimes \overline{\mathrm{CFT}}_{R}$, where the bar indicates space reflection. (We will actually restrict our discussion to nonchiral theories, for which $\overline{\mathrm{CFT}}_{R}=\mathrm{CFT}_{R}$.) The folded theory has two energy-momentum tensors $T_{L}$ and $\bar{T}_{R}$ that are separately conserved in the bulk, while only their sum $T_{\text {tot }} \equiv T_{L}+\bar{T}_{R}$ needs to be conserved at the boundary. What distinguishes interfaces from boundaries [and interface CFTs (ICFTs) from boundary CFTs (BCFTs)] is the existence of another, relative spin-2 current $T_{\text {rel }}=c_{R} T_{L}-c_{L} \bar{T}_{R}$, which measures the exchange of energy between left and right. Here $c_{L}$ and $c_{R}$

Published by the American Physical Society under the terms of the Creative Commons Attribution 4.0 International license. Further distribution of this work must maintain attribution to the author(s) and the published article's title, journal citation, and DOI. Funded by SCOAP ${ }^{3}$. are the central charges of the two CFTs. As usual, things simplify considerably in two dimensions. In this case, the transfer of energy across the interface is controlled by a single transmission or reflection coefficient $\mathcal{T}$ or $\mathcal{R}$, with $\mathcal{T}+\mathcal{R}=1$ [9-12]. The purpose of the present Letter is to derive a formula for these coefficients in the simplest holographic-interface model.

The model consists of two $\mathrm{AdS}_{3}$ slices separated by a string of tension $\sigma$. The $\mathrm{AdS}_{3}$ slices have radii $\ell_{L}$ and $\ell_{R}$, related to the CFT central charges by the Brown-Henneaux formula $c_{L, R}=3 \ell_{L, R} / 2 G$ [13], where $G$ is the threedimensional Newton's constant. Without loss of generality, we take $\ell_{L} \geq \ell_{R}$, so that the "false" higher-energy AdS vacuum is on the left, while the "true" AdS vacuum is on the right. For tensions inside the interval

$$
0 \leq \frac{1}{\ell_{R}}-\frac{1}{\ell_{L}} \leq 8 \pi G \sigma \leq \frac{1}{\ell_{R}}+\frac{1}{\ell_{L}}
$$

the string world sheet geometry is $\mathrm{AdS}_{2}$ corresponding to the ground state of the ICFT [4,14]. At the extremal values of the interval, the world sheet flattens out; i.e., the $\mathrm{AdS}_{2}$ radius diverges. The lower $\sigma$ limit in (1) corresponds to the Coleman-De Luccia bound [15], below which the false $\mathrm{AdS}_{3}$ vacuum is unstable to nucleation of bubbles. This is also the Bogomol'nyi-Prasad-Sommerfield bound for supergravity domain walls [16]. The upper limit, corresponds to the Randall-Sundrum fine-tuned tension, beyond which the string world sheet becomes de Sitter and gets anchored on a spacelike curve of the conformal boundary [4].

This model has been used as a toy model of holographic defects, including for calculations of holographic 
entanglement entropy (see, e.g., [17]). Here, we provide the first calculation of its transport properties. Our main result is the following expression for the energy-transmission coefficient defined in [9]:

$$
\mathcal{T}=\frac{4}{\ell_{L}+\ell_{R}}\left(\frac{1}{\ell_{L}}+\frac{1}{\ell_{R}}+8 \pi G \sigma\right)^{-1} .
$$

Together with the central charges, $\mathcal{T}$ was shown [9] to parametrize the most general two-point functions of energy-momentum tensors allowed by the symmetries of the problem.

As explained in [10,12], what was actually defined in [9] is the weighted-average transmission coefficient

$\mathcal{T}=\frac{c_{L} \mathcal{T}_{L}+c_{R} \mathcal{T}_{R}}{c_{L}+c_{R}}, \quad$ where $\mathcal{T}_{L, R}=\frac{\left(c_{L}+c_{R}\right) \mathcal{T}}{2 c_{L, R}}$

are the transmission coefficients for excitations incident on the interface from the left and right, respectively. Our formula for these directional transmission coefficients reads

$$
\mathcal{T}_{L, R}=\frac{2}{\ell_{L, R}}\left(\frac{1}{\ell_{L}}+\frac{1}{\ell_{R}}+8 \pi G \sigma\right)^{-1}
$$

The calculation of (2) and (4) is performed by scattering surface-gravity waves in a semiclassical geometry dual to the ground state of the ICFT. It relies on the usual condition of no outgoing waves at the Poincaré horizon, whose implementation we explain below.

Before we continue, let us comment on some salient features of our result. First, both $\mathcal{T}_{L}$ and $\mathcal{T}_{R}$ are monotonically decreasing functions of the tension $\sigma$. Their maximal and minimal values (in terms of the central charges) read $\mathcal{T}_{L, R}^{\max }=c_{R} / c_{L, R}$ and $\mathcal{T}_{L, R}^{\min }=c_{R, L} /$ $\left(c_{L}+c_{R}\right)$, or equivalently, for the average coefficients

$$
\begin{gathered}
\frac{2 c_{L} c_{R}}{\left(c_{L}+c_{R}\right)^{2}} \leq \mathcal{T} \leq \frac{2 c_{R}}{c_{L}+c_{R}} \\
\Leftrightarrow \frac{c_{L}^{2}+c_{R}^{2}}{\left(c_{L}+c_{R}\right)^{2}} \geq \mathcal{R} \geq \frac{c_{L}-c_{R}}{c_{L}+c_{R}} .
\end{gathered}
$$

The above lower bound on $\mathcal{R}$ is the same as the one following from the achronal averaged-null-energy condition (ANEC) in the ICFT [12], and it is stronger than the bound imposed by reflection positivity of the Euclidean theory [11], $\mathcal{R} \geq\left(c_{L}-c_{R} / c_{L}+c_{R}\right)^{2}$. This shows that reflection positivity does not necessarily imply the ANEC in ICFTs.

If the inequality $c_{L}>c_{R}$ is strict, both $\mathcal{T}_{L}$ and $\mathcal{T}$ are less than 1. Total transmission to signals incident from both sides is therefore only possible between degenerate $\mathrm{AdS}_{3}$ vacua separated by a tensionless string. This is the gravitational counterpart of a topological interface.
The opposite limit of total reflection $\mathcal{R} \rightarrow 1$ can only be reached by taking $c_{R} / c_{L} \rightarrow 0$, i.e., by depleting $\mathrm{CFT}_{R}$ of degrees of freedom, relative to $\mathrm{CFT}_{L}$. From the perspective of the false AdS vacuum, the string looks in this limit like the end-of-the-world brane of holographic BCFT $[18,19]$. As we will see from Eq. (8), this requires $G \ell_{L} \sigma$ to diverge. What is referred to as tension in $[18,19]$ is a finite leftover piece of $\sigma$. The total reflection limit in our model should be contrasted with the fact that, in more general ICFTs, factorizable interfaces can impose reflecting boundary conditions on each side for any values of $c_{L}, c_{R}$. In our minimal holographic model, however, the transmission of energy incident from the left can be shut down only if there are no degrees of freedom on the right side.

We should here stress that the transport coefficient $\mathcal{T}$ (or $\mathcal{R}$ ) and the boundary entropy (the logarithm of the $g$ factor) [20] are independent properties of a general CFT interface. This is illustrated by topological interfaces in free-field models, which can have arbitrarily large entropy [21,22] even though their transmission coefficient is always $\mathcal{T}=1$. The holographic duals of such interfaces were derived in $[23,24]$. Since topological interfaces can be deformed without changing the correlators of $T_{\mu \nu}$, we expect that the dual branes do not couple to gravity, in agreement with the analysis of the above references. We are thus led to conclude that tension is probably tied to $\mathcal{T}$ rather than entropy. This should be tested in more general holographic ICFT models where entanglement entropy and boundary entropy have been computed [17,25-28]. Note that, in the thin-brane holographic model that we are considering, these two quantities are in fact both fixed by the tension, since this is the only additional parameter associated with the brane. This situation is reminiscent of the case of the $a$ and $c$ central charges in four-dimensional CFT, which are equal in holographic models described by Einstein gravity, but not in general CFTs.

Holographic scattering states.-We describe now the main steps in the calculation of the reflection and transmission coefficients. As mentioned above, we use a minimal holographic model for the ICFT, consisting of two manifolds $M_{L, R}$ that are locally $\mathrm{AdS}_{3}$ and are joined on the world sheet of a tensile string. The asymptotic boundaries of these manifolds are the left (respectively, right) half planes glued along the CFT interface $P$. The latter extends in the bulk to surfaces $Q_{L} \subset M_{L}$ and $Q_{R} \subset M_{R}$ that are identified with each other and with the world sheet of the string, see Fig. 1. The gluing of $M_{L}$ to $M_{R}$ must obey the matching conditions [29]

$$
\begin{gathered}
\gamma_{L, \alpha \beta}=\gamma_{R, \alpha \beta}, \\
{\left[K_{\alpha \beta}\right]-[\operatorname{tr} K]_{\alpha \beta}=8 \pi G \sigma \gamma_{\alpha \beta},}
\end{gathered}
$$

where we have denoted by $\gamma_{L, R}$ and $K_{L, R}$ the induced metric and extrinsic curvature on $Q_{L, R}$, respectively, and we use 


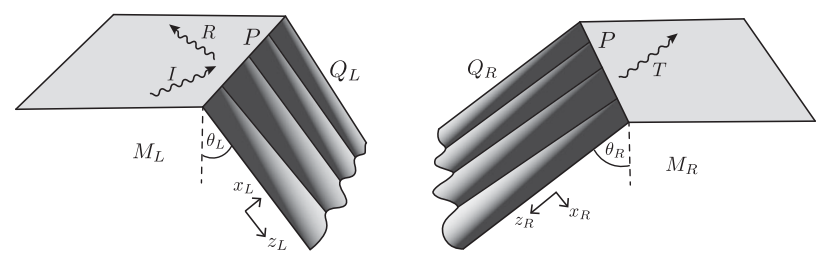

FIG. 1. Illustration of the holographic-interface geometry. The two spacetimes are glued together at the location of the world sheet $Q_{L} \equiv Q_{R}$. The interface $P$ is the intersection of the world sheet with the conformal boundary. The incident wave is denoted by $I$, and the reflected and transmitted waves are denoted by $R$ and $T$.

$[X] \equiv X_{R}-X_{L}$ to indicate discontinuities on the two sides of the interface. The extrinsic curvature in Eq. (6) is defined using a normal on the right side, which is oriented towards the right bulk (positive $x_{R}$ ), and a normal on the left side, which is oriented away from the left bulk (positive $x_{L}$ ).

The ICFT vacuum is described in Fefferman-Graham coordinates by the solution $[4,14]$

$$
\begin{array}{ll}
d s_{L}^{2}=\frac{\ell_{L}^{2}}{y_{L}^{2}}\left[d y_{L}^{2}+d u_{L}^{2}-d t_{L}^{2}\right] & \text { for } u_{L} \leq y_{L} \tan \theta_{L}, \\
d s_{R}^{2}=\frac{\ell_{R}^{2}}{y_{R}^{2}}\left[d y_{R}^{2}+d u_{R}^{2}-d t_{R}^{2}\right] & \text { for } u_{R} \geq-y_{R} \tan \theta_{R},
\end{array}
$$

where $0 \leq y_{L, R}<\infty$. The world sheet $u_{L}=y_{L} \tan \theta_{L}$ or $u_{R}=-y_{R} \tan \theta_{R}$ subtends an angle $\pi / 2+\theta_{L}$ or $\pi / 2+\theta_{R}$ to the left or right halves of the conformal interface, respectively. The world sheet metric is $\mathrm{AdS}_{2}$ with radius $\ell_{W}$ obeying

$$
\ell_{W}=\frac{\ell_{L}}{\cos \theta_{L}}=\frac{\ell_{R}}{\cos \theta_{R}}=\frac{\tan \theta_{L}+\tan \theta_{R}}{8 \pi G \sigma} .
$$

The first two equalities follow from (6a) and the last one from (6b). It will be later convenient to employ the rotated coordinates

$$
\begin{aligned}
& \left(\begin{array}{l}
u_{L} \\
y_{L}
\end{array}\right)=\left(\begin{array}{cc}
\cos \theta_{L} & \sin \theta_{L} \\
-\sin \theta_{L} & \cos \theta_{L}
\end{array}\right)\left(\begin{array}{l}
x_{L} \\
z_{L}
\end{array}\right), \\
& \left(\begin{array}{l}
u_{R} \\
y_{R}
\end{array}\right)=\left(\begin{array}{cc}
\cos \theta_{R} & -\sin \theta_{R} \\
\sin \theta_{R} & \cos \theta_{R}
\end{array}\right)\left(\begin{array}{l}
x_{R} \\
z_{R}
\end{array}\right),
\end{aligned}
$$

in which the unperturbed string sits at $x_{L}=x_{R}=0$, and its world sheet can be parametrized by $t_{L}=t_{R} \equiv t$ and $z_{L}=z_{R} \equiv z$.

In principle, one would like to solve the matching problem (6) for a generic metric and a fluctuating interface on the conformal boundary. It is, however, sufficient for our purposes to set all ICFT sources to zero, and only consider normalizable excitations of the fields. These are particularly simple in pure $\mathrm{AdS}_{3}$, where the most general solution of the Einstein equations in Fefferman-Graham coordinates can be written as [30-34]

$d s^{2}=\frac{\ell^{2} d y^{2}}{y^{2}}+\left[\frac{\ell^{2} g_{\alpha \beta}^{(0)}}{y^{2}}+g_{\alpha \beta}^{(2)}+\frac{y^{2}}{4 \ell^{2}} g_{\alpha \beta}^{(4)}\right] d w^{\alpha} d w^{\beta}$,

with $g^{(4)}=g^{(2)}\left(g^{(0)}\right)^{-1} g^{(2)}$ and, for flat boundary metric, $g_{\alpha \beta}^{(2)}=4 G \ell\left\langle T_{\alpha \beta}\right\rangle$. Here $\left\langle T_{\alpha \beta}\right\rangle$ is the vacuum expectation value (VEV) of the canonically normalized, traceless conserved energy-momentum tensor in some state of the dual CFT. Linearizing in the perturbation allows us to drop $g^{(4)}$, so that the correction to the standard $\mathrm{AdS}_{3}$ Poincare metric has arbitrary left- and right-moving waves, $g_{++}^{(2)}\left(w^{+}\right)$ and $g_{--}^{(2)}\left(w^{-}\right)$.

To reproduce the setup of Ref. [12], we consider a configuration with an incoming wave from the left, giving rise to a reflected wave on the left and a transmitted wave on the right. Explicitly, identifying the $w^{ \pm}$of (10) with $u \pm t$, and using monochromatic waves (working at the linearized level, the plane wave solutions can be superposed to wave packets), we have

$$
\begin{aligned}
{\left[d s^{2}\right]_{L}^{(2)}=} & 4 G \ell_{L} \epsilon\left[e^{i \omega\left(t_{L}-u_{L}\right)} d\left(t_{L}-u_{L}\right)^{2}\right. \\
& \left.+\mathcal{R}_{L} e^{i \omega\left(t_{L}+u_{L}\right)} d\left(t_{L}+u_{L}\right)^{2}\right]+ \text { c.c. }, \\
{\left[d s^{2}\right]_{R}^{(2)}=} & 4 G \ell_{R} \epsilon \mathcal{T}_{L} e^{i \omega\left(t_{R}-u_{R}\right)} d\left(t_{R}-u_{R}\right)^{2}+\text { c.c. },
\end{aligned}
$$

where $\mathcal{R}_{L}$ and $\mathcal{T}_{L}$ are the (a priori complex) relative amplitudes of the reflected and transmitted waves, and the subscript $L$ indicates that the incident wave came from the left. Anticipating the final result, we give the same names to these amplitudes as to the (real) reflection and transmission coefficients. In what follows, we will linearize our equations in the incoming flux $\left|\left\langle T_{--}\right\rangle\right|=\epsilon$.

Gluing $M_{L}$ with $M_{R}$ requires matching coordinates on the world sheet. We allow for this by writing $z_{L, R}=z+$ $\tilde{\epsilon} \zeta_{L, R}(z, t)$ and $t_{L, R}=t+\tilde{\epsilon} \lambda_{L, R}(z, t)$, where $z, t$ are the Poincaré coordinates of the $\mathrm{AdS}_{2}$ world sheet and we defined for convenience $\tilde{\epsilon}=4 G / \ell_{W} \epsilon$. Since we are keeping only linear order in $\epsilon$, we can set $t_{L}=t_{R}=t$ and $z_{L}=$ $z_{R}=z$ in the perturbation (11). The above changes of coordinates enter only through the expansion of the leading world sheet metric and extrinsic curvatures in (6). We also let $x_{L, R}=\tilde{\epsilon} \delta_{L, R}(z, t)$ be the fluctuating position of the string in the transverse dimension. (We use units where the metric is dimensionless; $t, x$, and $z$ have dimensions of length; $\epsilon$ and $\tilde{\epsilon}$ have dimensions of mass squared; and $\zeta_{L, R}, \lambda_{L, R}$, and $\delta_{L, R}$ have dimensions of length cubed.)

Thanks to time-translation invariance, we are allowed to work at fixed frequency

$$
\delta_{L, R}(z, t)=e^{i \omega t} \delta_{L, R}(z)+\text { c.c. }
$$

and similarly for $\zeta_{L, R}$ and $\lambda_{L, R}$. 
We have then six equations for the six functions $\delta_{L, R}$, $\zeta_{L, R}$, and $\lambda_{L, R}$. But common reparametrizations of the two charts are pure gauge, so only the transition functions

$$
\zeta \equiv \zeta_{L}-\zeta_{R} \quad \text { and } \quad \lambda \equiv \lambda_{L}-\lambda_{R}
$$

enter in the Eqs. (6). The problem may now look overconstrained, but two of the matching conditions (6b) are not actually independent. The reason is that all foliations of $\mathrm{AdS}_{3}$ obey the momentum constraints $D^{\alpha} K_{\alpha \beta}-D_{\beta} K=0$, where $D_{\alpha}$ is the covariant derivative with respect to the induced metric. Thus, once one of the conditions in Eq. (6b) has been solved, the other two are automatically satisfied up to constants. [With our fixed time dependence, the momentum constraint implies that the $z$ derivatives of two matching conditions vanish identically. In $D$ spacetime dimensions, a similar counting gives $(D-1)^{2}$ matching conditions for $D+1$ arbitrary functions. Hence, for $D>3$, two generic spacetimes cannot be matched.]

The brane fluctuations are induced by the gravity waves (11). The equations are more compact in terms of the combinations

$$
D \equiv \delta_{L}-\delta_{R}, \quad \Delta \equiv \tan \theta_{L} \delta_{L}+\tan \theta_{R} \delta_{R}-\zeta
$$

The four independent matching conditions read

$$
\begin{aligned}
\Delta+i \omega z \lambda & =z^{3}\left(\frac{\cos \theta_{L}}{2}(\mathbf{I}+\mathbf{R})-\frac{\cos \theta_{R}}{2} \mathbf{T}\right), \\
i \omega z \zeta-z \partial_{z} \lambda= & z^{3}\left(\sin \theta_{R} \cos \theta_{R} \mathbf{T}+\sin \theta_{L} \cos \theta_{L}(\mathbf{I}-\mathbf{R})\right), \\
z \partial_{z} \zeta+\Delta= & z^{3}\left(\frac{\sin ^{2} \theta_{R} \cos \theta_{R}}{2} \mathbf{T}-\frac{\sin ^{2} \theta_{L} \cos \theta_{L}}{2}(\mathbf{I}+\mathbf{R})\right), \\
z \partial_{z} D= & z^{3}\left(\frac{1}{i \omega z}(\mathbf{I}-\mathbf{R}-\mathbf{T})-\frac{\sin \theta_{L} \cos ^{2} \theta_{L}}{2}(\mathbf{I}+\mathbf{R})\right. \\
& \left.-\frac{\sin \theta_{R} \cos ^{2} \theta_{R}}{2} \mathbf{T}\right),
\end{aligned}
$$

where

$$
\begin{aligned}
\mathbf{I} & \equiv e^{-i \omega \sin \theta_{L} z}, \quad \mathbf{R}=\mathcal{R}_{L} e^{i \omega \sin \theta_{L} z}, \\
\mathbf{T} & \equiv \mathcal{T}_{L} e^{i \omega \sin \theta_{R} z}
\end{aligned}
$$

are the exponentials imprinted on the world sheet by the graviton waves (11). The first three equations are the matching conditions (6a), while the fourth is the $(t z)$ component of (6b), where we have used the second equation to simplify it. The three (almost) redundant matching conditions can be actually combined into an algebraic equation for $D$, so the integration constant in the last equation of (15) is fixed as in Eq. (18).

Consider first the homogeneous equations obtained by setting the right-hand sides in (15) to zero. The general solution reads

$$
\begin{aligned}
& -i \omega \lambda(z)=\frac{\Delta(z)}{z}=a_{+} e^{i \omega z}+a_{-} e^{-i \omega z}, \\
& -i \omega \zeta(z)=a_{+} e^{i \omega z}-a_{-} e^{-i \omega z}, \quad D=0 .
\end{aligned}
$$

The $z=0$ limit of these functions corresponds to sources in the dual ICFT. For instance $\delta_{L}(0)=\delta_{R}(0)$ is a source for the interface displacement operator. [Similarly, $\lambda(0)$ is the source for the dual operator that generates a relative reparametrization of the interface [35].] Linearizing in this source gives an $O\left(z^{-3}\right)$ correction to the induced metric. This is consistent with the fact that the scaling dimension of the displacement operator is $\mathfrak{D}=2$ [11]. In the absence of gravity waves, setting the sources to zero implies $a_{+}=a_{-}=0$. This shows that there are no normalizable states supported entirely by the interface.

The general solution to the linear inhomogeneous Eqs. (15), is given by (17) plus some special solution. After straightforward manipulations, we obtain

$$
\begin{aligned}
\frac{\Delta(z)}{z}= & \frac{1}{\omega^{2} \cos \theta_{L}}(\mathbf{I}+\mathbf{R})-\frac{1}{\omega^{2} \cos \theta_{R}} \mathbf{T}+a_{+} e^{i \omega z}+a_{-} e^{-i \omega z}, \\
\zeta(z)= & -\frac{\cos \theta_{L} z}{\omega^{2}}(\mathbf{I}+\mathbf{R})-\frac{i}{\omega^{3}}(\mathbf{I}-\mathbf{R})\left(\tan \theta_{L}+\frac{\sin \theta_{L} \cos \theta_{L}}{2} \omega^{2} z^{2}\right) \\
& -\frac{i}{\omega^{3}} \mathbf{T}\left(\tan \theta_{R}+i \cos \theta_{R} \omega z+\frac{\sin \theta_{R} \cos \theta_{R}}{2} \omega^{2} z^{2}\right)+\frac{i}{\omega}\left(a_{+} e^{i \omega z}-a_{-} e^{-i \omega z}\right), \\
\lambda(z)= & \frac{i}{\cos \theta_{L} \omega^{3}}(\mathbf{I}+\mathbf{R})\left(1-\frac{\cos ^{2} \theta_{L}}{2} \omega^{2} z^{2}\right)-\frac{i}{\cos \theta_{R} \omega^{3}} \mathbf{T}\left(1-\frac{\cos ^{2} \theta_{R}}{2} \omega^{2} z^{2}\right)+\frac{i}{\omega}\left(a_{+} e^{i \omega z}+a_{-} e^{-i \omega z}\right), \\
D(z)= & -\frac{i}{\omega^{3}}(\mathbf{I}-\mathbf{R})\left(1+\frac{\cos ^{2} \theta_{L}}{2} \omega^{2} z^{2}\right)+\frac{\sin \theta_{L} z}{\omega^{2}}(\mathbf{I}+\mathbf{R})+\frac{i}{\omega^{3}} \mathbf{T}\left(1-i \sin \theta_{R} \omega z+\frac{\cos ^{2} \theta_{R}}{2} \omega^{2} z^{2}\right) .
\end{aligned}
$$


Requiring that the sources vanish now gives

$$
D(0)=0 \Rightarrow \mathcal{R}_{L}+\mathcal{T}_{L}=1
$$

and further from $\zeta(0)=\lambda(0)=0$, we obtain

$$
\begin{aligned}
& a_{+}=\frac{1}{2 \omega^{2}}\left[\mathcal{T}_{L}\left(\frac{1+\sin \theta_{R}}{\cos \theta_{R}}+\tan \theta_{L}\right)-\frac{\left(1+\mathcal{R}_{L}\right)}{\cos \theta_{L}}\right], \\
& a_{-}=\frac{1}{2 \omega^{2}}\left[\mathcal{T}_{L}\left(\frac{1-\sin \theta_{R}}{\cos \theta_{R}}-\tan \theta_{L}\right)-\frac{\left(1+\mathcal{R}_{L}\right)}{\cos \theta_{L}}\right] .
\end{aligned}
$$

With these choices, all four functions are $O\left(z^{3}\right)$ near the conformal boundary and make $O(1)$ contributions to the world sheet metric, which can be interpreted as ICFT VEVs. This agrees again with the fact that the scaling dimension of the displacement operator is two [11].

Inserting the solution for $\delta_{L, R}$ in the expression for the induced metric shows that the latter is locally $\mathrm{AdS}_{2}$ (constant intrinsic Ricci curvature). Thus, as is the case for homogeneous $\mathrm{AdS}_{3} / \mathrm{CFT}_{2}$, here too the dynamics happens at the conformal boundary in spite of the presence of the string (dual to the conformal interface).

Up to this point, we have obtained a solution for the equations of motion of our model, which is valid for any value of $\mathcal{T}_{L}$. To proceed further, we have to make an assumption about the behavior of the solution at the Poincaré horizon. It is well known that, in the Lorentzian AdS/CFT correspondence, the boundary conditions at the conformal boundary do not determine the solution uniquely, because there are normalizable modes that vanish at the boundary and are regular in the interior [32]; this is the dual of the property that there are different Minkowskian propagators, depending on the choice of the initial state (retarded, advanced, Feynman, etc.).

The prescription of [36,37] (generalized by [38]), requires the absence of modes coming out of the horizon for the computation of a retarded correlator. In our case, it is not immediately obvious how to apply this prescription, since the problem is not formulated as the computation of causal response. One difficulty is that wave packets formed from (11) are localized in $u_{L, R}$ but not in the radial AdS coordinates $y_{L, R}$ and imprint superluminal waves on the functions $\delta_{L, R}, \zeta$, and $\lambda$ of the form $e^{i \omega t} \times(\mathbf{I}, \mathbf{R}$ or $\mathbf{T})$, see Eq. (16). But as illustrated by sea waves hitting an oblique seashore, these superluminal waves carry no energy. To see why, one must look at gauge-invariant quantities left unchanged by common reparametrizations of the two charts, $\delta \zeta_{L}=\delta \zeta_{R}$ and $\delta \lambda_{L}=\delta \lambda_{R}$. One such quantity, at the linearized order considered here, is the traceless part of the extrinsic curvature, which is continuous across the world sheet by Israel's matching condition (6b). A simple calculation gives

$$
\hat{K}_{ \pm \pm}=\frac{a_{ \pm} \omega^{2} \epsilon}{2 \pi \sigma \ell_{W}} e^{i \omega x^{ \pm}}+\mathcal{O}\left(\epsilon^{2}\right),
$$

where $x^{ \pm}=t \pm z$ and $\hat{K}_{\alpha \beta}$ denotes the traceless part of $K_{\alpha \beta}$. Here, the superluminal waves disappeared, and hence the "no-outgoing-wave" condition reduces to $a_{+}=0$. We would like to emphasize that this condition does not follow from the Israel junction equations but rather it is an extra condition imposed at the Poincaré horizon. Note also that the (discontinuous) trace parts, $K_{L, R}=$ $\pm 2 / \ell_{W} \tan \left(\theta_{L, R}\right)+\mathcal{O}\left(\epsilon^{2}\right)$, are not perturbed at linear order.

With the help of Eqs. (19) and (20), the no-outgoingwave condition implies

$$
\mathcal{T}_{L}=\frac{2 \cos \theta_{R}}{\cos \theta_{R}\left(1+\sin \theta_{L}\right)+\cos \theta_{L}\left(1+\sin \theta_{R}\right)} .
$$

Trading the angles for $\ell_{L, R}$ and $\sigma$ gives our result (4). It is nontrivial that $\mathcal{R}_{L}$ and $\mathcal{T}_{L}$, which started out as complex amplitudes in the gravitational-scattering problem, ended up as real, positive transport coefficients as required for a proper ICFT interpretation. This, together with the fact that our result obeys the nontrivial ANEC bound (5), is a strong a posteriori argument for its correctness.(One can check, e.g., that requiring $a_{-}=0$ leads to unphysical values for $\mathcal{T}$.)

Summary and outlook.-In this Letter, we evaluated the reflection and transmission from thin-brane holographic interfaces in $\mathrm{AdS}_{3}$. We found that the result (5) for the reflection coefficient is consistent with the lower ANEC bound, while its maximum approaches $\mathcal{R}=1$ only in the limit of infinite ratio of the central charges. This imperfect reflection might be a generic feature of holographic interfaces.

It would be interesting to study applications of our work in condensed-matter systems, as well as explore other holographic models, higher dimensions, and quantumgravitational corrections. Of special interest are the $1 / 2$ Bogomol'nyi-Prasad-Sommerfield holographic interfaces of $N=4$ super Yang-Mills $[39,40]$ and the associated topdown embedding of massive gravity [41]. Another important issue that will be discussed in a future publication [35] is universality, in particular, why $\mathcal{R}_{L, R}$ and $\mathcal{T}_{L, R}$ are independent of the nature of the incident wave as has been shown in the dual $\mathrm{CFT}_{2}$ [12].

It is also interesting to explore the relation of our work to the recent semiclassical computations of the Page curve $[42,43]$, where the relevant toy models of black holes coupled to heat baths were argued to admit doubly holographic BCFT or ICFT realizations [44-48]. Our results on reflection and transmission could perhaps come to use when coupling the black hole to the bath.

In this context, it has been pointed out that the transmission of energy across an interface differs from the transmission of information. It would be interesting to compare our results to various information theoretic 
measures and their dynamics in the presence of defects (see, e.g., [17,26,27,49-52]).

We would like to thank Denis Bernard, Damian Galante, Oleksandr Gamayun, Christopher Herzog, Diego Hofman, Donald Marolf, Marco Meineri, Yaron Oz, Vassilis Papadopoulos, Joao Penedones, and Massimo Porrati for valuable comments and discussions. D. G. is grateful for the KITP-UCSB graduate fellowship program, where part of this work was carried out. This research was supported in part by the Heising-Simons Foundation, the Simons Foundation, and National Science Foundation Grant No. NSF PHY-1748958. S. C. acknowledges the support of the ERC consolidator grant QUANTIVIOL awarded to Ben Freivogel.

*costas.bachas@phys.ens.fr

†s.chapman@uva.nl

*ge@lpt.ens.fr

\$policast@lpt.ens.fr

[1] E. Wong and I. Affleck, Tunneling in quantum wires: A boundary conformal field theory approach, Nucl. Phys. B417, 403 (1994).

[2] M. Oshikawa and I. Affleck, Boundary conformal field theory approach to the critical two-dimensional Ising model with a defect line, Nucl. Phys. B495, 533 (1997).

[3] A. Karch and L. Randall, Open and closed string interpretation of SUSY CFT's on branes with boundaries, J. High Energy Phys. 06 (2001) 063.

[4] A. Karch and L. Randall, Locally localized gravity, J. High Energy Phys. 05 (2001) 008.

[5] M. Porrati, Mass and gauge invariance: Holography for the Karch-Randall model, Phys. Rev. D 65, 044015 (2002).

[6] O. DeWolfe, D. Z. Freedman, and H. Ooguri, Holography and defect conformal field theories, Phys. Rev. D 66 , 025009 (2002).

[7] C. Bachas, J. de Boer, R. Dijkgraaf, and H. Ooguri, Permeable conformal walls and holography, J. High Energy Phys. 06 (2002) 027.

[8] D. Bak, M. Gutperle, and S. Hirano, A dilatonic deformation of $\mathrm{AdS}_{5}$ and its field theory dual, J. High Energy Phys. 05 (2003) 072.

[9] T. Quella, I. Runkel, and G. M. Watts, Reflection and transmission for conformal defects, J. High Energy Phys. 04 (2007) 095.

[10] T. Kimura and M. Murata, Transport process in multijunctions of quantum systems, J. High Energy Phys. 07 (2015) 072.

[11] M. Billó, V. Gonçalves, E. Lauria, and M. Meineri, Defects in conformal field theory, J. High Energy Phys. 04 (2016) 091.

[12] M. Meineri, J. Penedones, and A. Rousset, Colliders and conformal interfaces, J. High Energy Phys. 02 (2020) 138.

[13] J. Brown and M. Henneaux, Central charges in the canonical realization of asymptotic symmetries: An example from three dimensional gravity, Commun. Math. Phys. 104, 207 (1986).
[14] C. Bachas, in Proceedings, Meeting on Strings and Gravity: Tying the Forces Together, edited by M. Henneaux and A. Sevrin (Francqui Scientific Library, Brussels, 2003), pp. 9-17.

[15] S. R. Coleman and F. De Luccia, Gravitational effects on and of vacuum decay, Phys. Rev. D 21, 3305 (1980).

[16] M. Cvetic, S. Griffies, and S.-J. Rey, Static domain walls in $N=1$ supergravity, Nucl. Phys. B381, 301 (1992).

[17] T. Azeyanagi, A. Karch, T. Takayanagi, and E. G. Thompson, Holographic calculation of boundary entropy, J. High Energy Phys. 03 (2008) 054.

[18] T. Takayanagi, Holographic Dual of a Boundary Conformal Field Theory, Phys. Rev. Lett. 107, 101602 (2011).

[19] M. Fujita, T. Takayanagi, and E. Tonni, Aspects of AdS/BCFT, J. High Energy Phys. 11 (2011) 043.

[20] I. Affleck and A. W. W. Ludwig, Universal Noninteger "Ground-State Degeneracy" in Critical Quantum Systems, Phys. Rev. Lett. 67, 161 (1991).

[21] J. Fuchs, M. R. Gaberdiel, I. Runkel, and C. Schweigert, Topological defects for the free boson CFT, J. Phys. A 40, 11403 (2007).

[22] C. Bachas and I. Brunner, Fusion of conformal interfaces, J. High Energy Phys. 02 (2008) 085.

[23] J. R. Fliss, X. Wen, O. Parrikar, C.-T. Hsieh, B. Han, T. L. Hughes, and R. G. Leigh, Interface contributions to topological entanglement in abelian Chern-Simons theory, J. High Energy Phys. 09 (2017) 056.

[24] M. Gutperle and J. D. Miller, Topological interfaces in Chern-Simons theory and the $\mathrm{AdS}_{3} / \mathrm{CFT}_{2}$ correspondence, Phys. Rev. D 99, 026014 (2019).

[25] M. Chiodaroli, M. Gutperle, and L.-Y. Hung, Boundary entropy of supersymmetric Janus solutions, J. High Energy Phys. 09 (2010) 082.

[26] K. Jensen and A. O'Bannon, Holography, entanglement entropy, and conformal field theories with boundaries or defects, Phys. Rev. D 88, 106006 (2013).

[27] J. Erdmenger, M. Flory, and M.-N. Newrzella, Bending branes for DCFT in two dimensions, J. High Energy Phys. 01 (2015) 058.

[28] M. Gutperle and J. D. Miller, Entanglement entropy at holographic interfaces, Phys. Rev. D 93, 026006 (2016).

[29] W. Israel, Singular hypersurfaces and thin shells in general relativity, Nuovo Cimento B 44, 1 (1966); , Erratum, Nuovo Cimento B 48, 463(E) (1967).

[30] K. Skenderis and S. N. Solodukhin, Quantum effective action from the AdS/CFT correspondence, Phys. Lett. B 472, 316 (2000).

[31] M. Banados, Three-dimensional quantum geometry and black holes, AIP Conf. Proc. 484, 147 (1999).

[32] V. Balasubramanian, P. Kraus, and A. E. Lawrence, Bulk versus boundary dynamics in anti-de Sitter spacetime, Phys. Rev. D 59, 046003 (1999).

[33] M. Rooman and P. Spindel, Uniqueness of the asymptotic $\mathrm{AdS}_{3}$ geometry, Classical Quantum Gravity 18, 2117 (2001).

[34] K. Krasnov, On holomorphic factorization in asymptotically AdS 3D gravity, Classical Quantum Gravity 20, 4015 (2003).

[35] C. Bachas, S. Chapman, D. Ge, and G. Policastro (to be published).

[36] D. T. Son and A. O. Starinets, Minkowski-space correlators in AdS/CFT correspondence: recipe and applications, J. High Energy Phys. 09 (2002) 042. 
[37] C. Herzog and D. Son, Schwinger-Keldysh propagators from AdS/CFT correspondence, J. High Energy Phys. 03 (2003) 046.

[38] K. Skenderis and B. C. van Rees, Real-time gauge/gravity duality: prescription, renormalization and examples, J. High Energy Phys. 05 (2009) 085.

[39] E. D’Hoker, J. Estes, and M. Gutperle, Exact half-BPS type IIB interface solutions I: local solution and supersymmetric Janus, J. High Energy Phys. 06 (2007) 021.

[40] E. D'Hoker, J. Estes, and M. Gutperle, Exact half-BPS type IIB interface solutions II: flux solutions and multi-Janus, J. High Energy Phys. 06 (2007) 022.

[41] C. Bachas and I. Lavdas, Massive Anti-de Sitter gravity from string theory, J. High Energy Phys. 11 (2018) 003.

[42] G. Penington, Entanglement wedge reconstruction and the information paradox, J. High Energy Phys. 09 (2020) 002.

[43] A. Almheiri, N. Engelhardt, D. Marolf, and H. Maxfield, The entropy of bulk quantum fields and the entanglement wedge of an evaporating black hole, J. High Energy Phys. 12 (2019) 063.

[44] A. Almheiri, R. Mahajan, J. Maldacena, and Y. Zhao, The Page curve of Hawking radiation from semiclassical geometry, J. High Energy Phys. 03 (2020) 149.
[45] H. Z. Chen, Z. Fisher, J. Hernandez, R. C. Myers, and S.-M. Ruan, Information flow in black hole evaporation, J. High Energy Phys. 03 (2020) 152.

[46] M. Rozali, J. Sully, M. Van Raamsdonk, C. Waddell, and D. Wakeham, Information radiation in BCFT models of black holes, J. High Energy Phys. 05 (2020) 004.

[47] H. Geng and A. Karch, Massive islands, J. High Energy Phys. 09 (2020) 121.

[48] H. Z. Chen, R. C. Myers, D. Neuenfeld, I. A. Reyes, and J. Sandor, Quantum extremal islands made easy, part I: Entanglement on the brane, arXiv:2006.04851.

[49] K. Sakai and Y. Satoh, Entanglement through conformal interfaces, J. High Energy Phys. 12 (2008) 001.

[50] M. Gutperle and A. Trivella, Note on entanglement entropy and regularization in holographic interface theories, Phys. Rev. D 95, 066009 (2017).

[51] B. Czech, P. H. Nguyen, and S. Swaminathan, A defect in holographic interpretations of tensor networks, J. High Energy Phys. 03 (2017) 090.

[52] S. Chapman, D. Ge, and G. Policastro, Holographic complexity for defects distinguishes action from volume, J. High Energy Phys. 05 (2019) 049. 